\title{
Assessing the field of health policy and systems research using symposium abstract submissions and machine learning techniques
}

\begin{abstract}
The field of health policy and systems research (HPSR) has grown rapidly in the past decade. Examining recently aggregated data from the Global Symposia on Health Systems Research, a key global fora for HPSR convened by the largest international society—Health Systems Global (HSG)—provides opportunities to enhance existing research on HPSR capacity using novel analytical techniques. This addresses the demand not only to map the field but also to examine potential predictors of acceptance to, and participation at, these global conferences to inform future work and strategies in promoting HPSR. We examined data from the abstracts submitted for two Global Symposia on Health Systems Research in 2016 and 2018 by type of institution, countries, regional groupings, and gender. After mapping hotspot areas for HPSR production, we then examined how the corresponding author's characteristics were associated with being accepted to present at the Global Symposia. Our findings showed that submissions for the Global Symposia increased by 12\% from 2016 to 2018. Submissions increased across all participant groups, particularly the for-profit organizations and research/consultancy firms showing the highest increases, at $58 \%$ for both. We also found reduced submissions from high-income countries, while submissions from LMICs, Sub-Saharan Africa and Latin America, increased substantially revealing inclusivity values of symposium organizers. Submissions increased to a larger extent among women than men. Being a woman, coming from a high income country, and having multiple abstracts submitted were found to be significant predictors for an abstract to be accepted and presented in the Symposia. Findings provide critical baseline information on the extent of interest and engagement in a global forum of various institutions and researchers in HPSR that can be useful for setting future directions of HSG and other similar organizations to support the advancement of HPSR worldwide.
\end{abstract}




\section{Background}

3 Over the past decade, health systems strengthening (HSS) emerged as a priority for most organizations

4 involved in global health given concerns that disease-focused investments have delivered slow progress on

5 the health Millennium Development Goals (MDGs) and on health systems (Hafner and Shiffman, 2013).

6 This led to an emerging consensus that without robust, responsive, and efficient health systems, the

7 Sustainable Development Goals (SDGs) cannot be achieved, and Universal Health Coverage (UHC)

8 aspirations could not be met. Consequently, there has been a surge in activities around health system

9 strengthening (Hafner and Shiffman, 2013). In response to this demand, the thematic area of Health Policy

10 and Systems Research (HPSR) emerged drawing on a range of disciplines and demonstrating an

11 unprecedented level of interest (Bennett et al., 2011). HPSR is both a multidisciplinary and interdisciplinary

12 field identified by its topics of research and scope of questions asked, including international, national and

13 local health systems and their interconnectivities, and policies made and implemented at all levels of the

14 health system (Sheikh et al., 2011). In 1996, WHO published the volume Health Policy and Systems

15 Development: an Agenda for Research, (WHO, 1996) laying the foundation for the establishment of the

16 Alliance for HPSR, (AHPSR, 2018) an international partnership hosted by the WHO, in 1999 (Bennett et

17 al., 2018). With strong leadership within the WHO, the Alliance for HPSR harmonized efforts to better engage policymakers and practitioners in HPSR, increasing focusing on HPSR. Such growing interest in the field, along with the 2000 World Health Report, (WHO, 2013a) the 2003 Task Force for Health Systems Research, the 2004 Mexico Statement on Health Research, (The Mexico Statement on Health Research, 2004), among others, intensified interest in health systems research, calling for greater investments in the

22 field. See Appendix 1 for a list of statements and publications relevant to HPSR. HPSR has since evolved

23 from both country interests and global level processes (Bennett et al., 2018). These recent developments in

24 HSS and HPSR also led to the WHO strategy on health policy and systems research (2012), the First World

25 Report on Health Policy and Systems Research (WHO, 2013b), and a call by health policy and systems

26 researchers for a common platform to share recent evidence and tools to further advance the field. These

27 developments led to the Global Symposium on Health Systems Research, which was convened first in 2010

28 (Kraushaar et al., 2012) and every two years thereafter, with Health Systems Global becoming established

29 in 2011 as the first international membership organization fully dedicated to promoting health systems

30 research and knowledge translation.

32 HSG became one of the organizations to spearhead the development of HPSR as a professional field and 33 engage a wide range of actors (e.g. researchers, policy makers, civil society, media) and from Global North 34 and South to ensure that the latest research aligns with national and global priorities to best inform policy 
1 that leads to health systems transformation (Kraushaar et al., 2012). (AHPSR, 2018) The HSG Strategic

2 Plan (HSG, 2016a) further emphasized that HSG should be driven by engagement and participation of its

3 diverse membership and should foster equity, diversity and inclusiveness to be able to deliver on its mission

4 to connect and engage researchers, policymakers, health care managers, educators, civil society, the media,

5 and donors from around the world to advance the field of HPSR and ensure that the knowledge required to

6 strengthen health systems is generated and used (HSG, 2016a). A core aspiration of HSG is to support

7 initiatives to increase capacity of individuals and institutions involved in HPSR, often realized through

8 participation in the Global Symposia on Health Systems Research, which have become the most important

9 fora for HPSR sharing and learning.

11 Thus, similar to other global health organizations, inclusivity and diversity are critical to the Global

12 Symposia on Health Systems Research to ensure that no stakeholders with ability and interest to contribute

13 to HPSR, are excluded from the global discourse on health systems development. Like other major health

14 conferences, participation in the conference is through abstract submissions and selection procedures.

15 Hence, expanding participation and meeting the HSG's inclusivity and diversity objectives requires having

16 an abstract accepted after a blind and independent evaluation by peers. However, to date, there is no

17 evidence on how abstract and author characteristics may be associated with acceptance into the Global

18 Symposia. Examining these associations is critical to ensure adequate representation of key health system

19 actors, reduce any potential selection bias on whose voices get to be heard in such global conferences,

20 monitor the diversity of participants and strategize how inclusivity and diversity can be further

21 operationalized in future global symposia and other research fora. Realizing any gaps in diversity and

22 inclusivity early on is critical to understand who shapes the HPSR discourse and how representative current

23 findings were of the voices of the different health system actors from diverse parts of the world.

Apart from ensuring representation of key health system actors, there is also a need to ensure inclusivity across countries. Since HSS is a long-term iterative process in which local stakeholders, rather than donors or external experts, take the lead in adapting evidence-based solutions to local political and cultural contexts (Sundewall et al., 2011), promoting locally generated research is important to inform critical policy decisions. HPSR benefits from being embedded within a particular context and close engagement with local actors (Bennett et al., 2011; Gilson et al., 2017). Therefore, it needs to be driven by local actors who have an intimate understanding of their own health systems and the challenges that they face (WHO, 2017).

32 There have been a range of HPSR capacity assessments, (Gonzalez Block and Mills, 2003; Gonzalez-Block, 33 2004; Block, 2006) however, evidence examining the institutional and individual characteristics of the 34 actors in the field has been less common particularly applying innovative analytical methods such as 
1 machine learning to generate additional information and using of data other than bibliometrics to fill this

2 gap (Sheikh et al., 2016).

4 We seek to address the lack of recent evidence on who shapes the HPSR field, what countries are most

5 involved, how inclusivity in the Global symposium is achieved and how the HSPR field is evolving, by

6 analyzing a newly aggregated dataset on submissions and participation from the past two global conferences

7 on health systems research held in 2016 and 2018. Since HSG is the largest organization in the field and

8 assuming that an online abstract submission system has a wider reach and is more accessible to those

9 interested in it, we used the HSG abstract submission database as an way of assessing the growth in interest

10 and engagement in the field of HPSR. We explore the following three questions: First, who submits

11 abstracts to the Global Symposia and how did this evolve between 2016 and 2018? Second, how does

12 acceptance of abstracts into the symposium programme vary by author characteristics, and how has this

13 evolved? Third, how does acceptance of abstracts vary by geographical locations? Answering these

14 questions allows us to offer a comprehensive snapshot of the participants in the field using the symposia

15 dataset as critical additional information to assess the reach of HPSR globally.

\section{Methods}

19 Data sources

20 We used data extracted from the individual abstracts submitted for either oral or poster presentations for 21 the two Global Symposia organized by Health Systems Global in 2016 and 2018. All submissions were

22 collected by Health Systems Global and relevant information on author's first name, affiliation, location,

23 and abstract submission result (oral, poster, rejected) were extracted from the online submission system.

24 All extracted data were then anonymized and made available as a dataset. About a year before each

25 Symposium, a call for abstracts is launched that invites different health system stakeholders to submit an

26 abstract either for oral or poster presentation, and for organized sessions that include several presenters.

27 The total organized and individual abstracts submitted is 3016 in 2016 and 3364 in 2018. Every

28 Symposium, individual abstracts accounted for $85 \%$ of the total abstracts submitted. This analysis uses

29 only data from the individual abstracts that were submitted online because we do not have complete

30 information collected for organized session submissions. Furthermore, individual abstracts were seen as

31 more representative of the global HPSR field and they also allow capturing detailed information about the

32 corresponding authors such as first name, institutional and country affiliation. Since only the

33 corresponding author's first names were extracted from the database, data are not identifiable. 


\section{Outcomes}

2 Our outcome variables were the number of submitted abstracts, and the proportion of acceptances for oral

3 or poster presentation among submitted abstracts. We then determined the associations between the total

4 number of submitted and accepted abstracts and the corresponding author's institutional affiliation,

5 gender, and country of location.

\section{Independent variables}

8 Our exposure variables were the characteristics of the corresponding author including the type of their 9 institutional affiliation, gender, and their location. First, the institutions were classified as: academic 10 institution/university, non-profit organization, government, research firms and consultancies, bilateral and 11 multilateral agencies, and for-profit organizations and others. The author's country location was

12 categorized according to the World Bank country income category (low, low-middle, upper-middle, and

13 high income) and regional groupings (East Asia and the Pacific, Europe and Central Asia, Latin America

14 and the Caribbean, Middle East and North Africa, North America, South Asia, and Sub-Saharan Africa).

15 We also explored total number of abstracts originating from a given institution, as an independent 16 variable.

\section{Statistical analysis}

19 We calculated summary statistics for the corresponding authors' types of institutional affiliation, gender, 20 country and total number of abstracts submitted by the affiliated institution. First, types of institutional

21 affiliation were identified by extracting keywords from the institutional affiliations of the corresponding

22 authors. We then categorized all the keywords extracted from each institutional affiliation of the

23 corresponding authors and classified them into themes that became the types of institutional affiliations.

24 Each institutional affiliation was then coded and categorized into the key themes identified. Second, since

25 gender was not captured in the online abstract submission system, the gender was identified using the

26 "Genderize.io" software package (Genderize, 2018) from Python (Python, 2018) that predicts the gender

27 of the corresponding author based on their first name and country affiliation. The "Genderize.io" package

28 determines the gender of a first name using Python's application programming interfaces that have big

29 datasets about user profiles from across major social networks. These profiles from major social networks

30 that include the first name, gender and country were then matched into the list of first names and

31 countries in our data to predict whether the first name is a man or a woman, based on the country attached

32 to the corresponding author. In cases where data from major social networks from Genderize.io could not

33 be matched to the first name and country of the corresponding author ( $<10 \%$ of the data), we used

34 LinkedIn to search for the most likely gender of the corresponding author. Specifically, we assigned male 
1 or female gender to the corresponding author using LinkedIn's search results, matching the gender of

2 those who had a similar first name and were working in the health policy and systems research area.

3 Health Systems Global has more than 10,000 connections in LinkedIn, which allowed us to search for

4 other health policy and systems researchers. Third, the locations of the corresponding authors were

5 mapped by geocoding the institutional affiliation, city, and country data provided in the online abstract

6 submission system. Using ArcGIS version 10.6.1, we geocoded the institutional affiliations which

7 provided geographical coordinates corresponding to the location of the authors. Countries of

8 corresponding authors were then classified into the World Bank income categories and regional

9 groupings.

11 After calculating summary statistics for the corresponding authors, we then calculated the number of

12 abstracts that were submitted for 2016 and 2018 and computed the percent change in abstract submissions

13 and acceptance rates based on the corresponding authors' characteristics. We mapped both the number of

14 abstracts submitted and accepted to the Global Symposia to highlight areas of lowest and highest

15 participation in the global conference, as well as to identify countries that did not participate in either the

162016 or 2018 Global Symposium. Lastly, using data pooled across the two Symposia, we used

17 multivariate regressions and modeled how abstract acceptance for either an oral or a poster presentation

18 was associated with the different corresponding author characteristics: institutional type, gender, country

19 income group, and geographic region. All p values were adjusted for multiple comparisons testing. Apart

20 from gender predictions using Python version 3.7.0 and geocoding of institutions using ArcGIS version

21 10.6.1, all other analyses were done in Stata version 15. All maps were plotted using the Database of

22 Global Administrative Areas and produced using ArcGIS version 10.6.1.

23

24 Role of the funding source

25 The authors did not receive funding for this work. The Global Symposia on Health Systems Research are

26 funded by donors listed on the Symposium website (HSG, 2016b, 2018a). The funders had no role in

27 study design, data collection and analysis, decision to publish, or preparation of the manuscript. The

28 corresponding author had access to anonymized data in the study and had final responsibility for the

29 decision to submit for publication.

31 Results

32

33 Abstract submissions by author characteristics. A total of 5,447 abstracts were submitted for the

34 Global Symposium on Health Systems Research in 2016 and 2018 (Table 1). From 2016 to 2018, we 
1 found a $12 \%$ increase in abstract submissions ( $n=2,572$ in 2016 and 2,875 in 2018). While we did not see

2 a significant increase between the two Symposia in the number of abstracts originating from academic

3 institutions, we observed growth in submissions from all other types of institutions (Table 1), with the

4 highest growth observed among for-profit organizations and research and consulting firms (58\%) and

5 non-profit organizations (40\%) followed by governmental institutions (35\%). Across the country income

6 categories, high-income countries' submissions declined by $3 \%$, while submissions increased by $29 \%$,

$723 \%$ and $15 \%$ respectively for low-income, lower-middle and upper-middle income countries. Increases

8 in abstract submissions were also particularly prominent in Sub-Saharan Africa (39\%), followed by Latin

9 America and the Caribbean (31\%) and South Asia posting lowest growth (5\%) (Table 1). Decreases were

10 seen for North America and East Asia and the Pacific, at 17\% and 7\% reduction, respectively. We also

11 found that there were more abstract submissions from women than men in both years of the Global

12 Symposia with men's submissions increasing by 9\% and women's increasing by $15 \%$ between 2016 and

132018.

14

15

16

17

\section{[Table 1]}

Institutional engagement. In total, out of the 1,841 institutions from 127 countries that submitted an abstract for both years of the global conference, 1517 (82\%) are institutions that submitted only for either 2016 or 2018 while 324 (18\%) comprise institutions that submitted abstracts in both 2016 and 2018 (Table 2). In 2016, there were abstracts submitted from 846 institutions from 105 countries. In 2018, the number of institutions increased to 995 institutions from 116 countries. Among these institutions that submitted an abstract, 500 institutions were represented by only one abstract in 2016, which further increased to 582 institutions submitting only one abstract in 2018. The highest number of submissions for each Symposia were from one institution accounting for 3\% of the total submissions in 2016 and two institutions accounting for $5 \%$ of the total submissions in 2018. The mean number of submitted abstracts per institution declined from 26 abstracts (95\% CI: 24.2-26.97) in 2016 to 23 abstracts in 2018 (95\% CI:21.9-24.4), the median being 11 and 9 abstract submissions, respectively (Table 1). Although the number of institutions submitting an abstract increased from 2016 to 2018, the number of institutions who had an accepted abstract decreased from 507 institutions in 2016 to 475 institutions in 2018 (Appendix 2) and the median number of abstracts accepted from an institution increased from 5 to 6 abstracts (Table 1). Appendix 3 specifies where the institutions of the corresponding authors were located.

[Table 2]

Country engagement. Figure 1 and 2 maps the number of abstracts submitted and accepted per country for the two Symposia, indicating the absence of participation from some countries mostly in the former Soviet states and some parts of Africa. However, six countries (Armenia, Belarus, Kazakhstan, Kyrgyz 
1 Republic, Moldova and Tajikistan) from Former Soviet Union that did not submit to HSR2016 submitted

2 abstracts to HSR2018. However, none of these submissions from these countries were accepted.

[Figures 1 and 2]

Abstract submissions versus abstract acceptance. Of the 2,572 abstracts submitted in 2016, 43.3\% were accepted. On average, $10 \%$ of the total abstracts are accepted for oral presentation, $31 \%$ for poster

8 presentations, and 59\% are not accepted across the two time periods. This acceptance rate decreased to

$937.8 \%$ in 2018. (Table 3). Although the overall acceptance rate declined by $5.5 \%$ between the two

10 Symposia, the number of accepted abstracts were relatively similar at 1,114 in 2016 and 1,087 in 2018.

Abstract acceptance by author characteristics. For the two Symposia, the number of abstracts submitted and accepted were higher for women than men (Table 3). Acceptance rate was highest for multilateral and bilateral agencies (51\% in 2016 and 40\% in 2018), followed by research and consulting firms (50\% in 2016 and 39\% in 2018) and government agencies (44\% in 2016 and 29\% in 2018). Forprofit organizations had the highest reduction in acceptance rates at 15\%. Although there were more submissions from Latin America and the Caribbean and Sub-Saharan Africa across the two symposia, these regions also had more rejections, with acceptance rates decreasing by $16 \%$ and $12 \%$ respectively. Although submissions were also lower for high-income countries in 2018 compared to 2016, acceptance rate for high-income countries increased by $2 \%$ between the Symposia. In summary, bivariate associations showed that gender, institutional type, region, and country income group affiliations were

22 significantly associated with having an abstract accepted or not for the 2018 Symposia ( $\mathrm{p}=0.001$ for all),

23 while only country income group was significantly associated with abstract acceptance for the 2016

24 Symposia $(\mathrm{p}=0.024)$.

\section{Associations between abstract acceptance and author characteristics across the two Global}

28 Symposia. Our multivariate regression models examined the associations between abstract acceptance and author characteristics (Table 4). Model 1 reveals that being female OR=1.22 (95\% CI:1.09 - 1.36) and coming from a high-income country significantly increase the odds of having an abstract accepted.

31 Moreover, the higher the number of abstracts submitted by the corresponding author's institution, the 32 higher were the odds of being accepted OR=1.003 (95\% CI:1.002-1.005). While the concentration of 33 abstracts with institutions increases the acceptance rate, the association $(\mathrm{OR}=1.003)$ is marginal. Adding

34 other author characteristics such as the type of institutional (Model 2) and regional affiliation of an author 
1 (Model 3) did not change the significance of gender, country income grouping, and number of abstracts

2 submitted per institution in having an abstract accepted for the Symposia. When considering the year of

3 submission, authors submitting abstracts to HSR2018 had a lower chance of being accepted OR=0.81

4 (95\% CI: 0.7-0.9) than those who submitted to HSR2016, controlling for all other author characteristics. 5

[Table 4]

\section{Discussion}

10 To our knowledge, this is the first study that sought to examine the diversity and inclusivity of the field of 11 HPSR by exploring participation in the two largest Global Symposia on Health Systems Research-in

122016 and 2018 - and to identify recent trends in HPSR using newly available data from Health Systems

13 Global. Specifically, it traced the growth of the field using abstract submissions and acceptance rates as

14 potential indicators, as well as author characteristics as determinants of engagement in and inclusivity of

15 the field. Our findings suggest increasing interest in participating in Global Symposium with a 12\%

16 increase in submissions for the Global Symposia from 2016 to 2018, including greater interest by non-

17 academic institutions. Submissions increased across all types of institution demonstrating involvement of

18 diverse stakeholders especially those outside academia, while academic submission remained relatively

19 stable (0.1\% increase). We also found that submissions from lower-income countries have shown larger

20 increases while submissions from high-income countries have declined by $3 \%$. We also noted that certain

21 institutions and countries may have higher concentration of abstract submissions, posing challenges to

22 HSG in ensuring inclusivity and diversity in future Symposia. Specifically, a substantial increase in

23 submissions was also noted for the Sub-Saharan Africa and Latin America and the Caribbean regions.

24 However, engagement from some other regions (North America and East Asia and the Pacific) declined.

25 Although submissions increased, a decline was noted in the number of institutions that had abstracts

26 accepted. Importantly, the reach of HPSR seems to have improved, with a larger number of institutions

27 submitting at least one abstract, indicating a growing interest in HPSR and increased institutional

28 diversity.

30 Our findings on the increased number of abstracts submitted for the two Global Symposia indicate an

31 increasing engagement in the field of HPSR, particularly across all types of institutions and in low- and

32 middle- income countries. Such expansion is in line with the HSG's vision and Strategic Plan (HSG,

33 2016a) to increase engagement across all sectors and especially by the Global South. Several pre-

34 Symposia strategies may have contributed to this growth. For example, HSG strengthened their 
1 communication portfolio, particularly their online presence through the HSG website, blogs (HSG,

2 2018b, 2018c) and other social media channels. HSG also prioritized better engagement with HSG

3 members through co-organizing pre-Symposia activities with the HSG's Thematic Working Groups

4 (TWG) and Symposium co-organizers to encourage more people to participate at the Symposia. A

5 number of regional events may have also played a role in increasing submissions. For example, pre-

6 Symposia meetings were held in Latin America and Caribbean (HSG, 2018d) and a webinar was

7 organized specifically targeted for Africa-based members to increase their region's participation (HSG,

8 2018e). These and other regionally targeted strategies may have led to the observed increases in abstract

9 submissions observed in Latin America and the Caribbean and Africa. However, the low level of

10 participation from some parts of Africa, the Middle East and Central and especially the eastern part of the

11 European region (Figure 2) may suggest that HPSR capacity is still weak in these sub-regions and HSG

12 and its members should focus on supporting institutions and engaging more stakeholders from these

13 regions.

15 Nonetheless, this growth has had some negative implications. Thus, the probability of abstract acceptance

16 declined by $19 \%$ between the two Symposia. The regression analysis indicates that if an author is not

17 from a high-income country, they may face lower chances of having an abstract accepted for the

18 Symposia, suggesting the need to further strengthen support for the field of HPSR in lower-income

19 country settings. However, while competition for abstract acceptance is growing, the Symposium

20 organisers have elected to keep the overall number of sessions constant. The number of sessions

21 available is determined by the conference duration (traditionally 3 days of the main programme) and the

22 number of sessions that run in parallel (itself constrained by the number of rooms available at the

23 conference venue). Both Symposia venues were able to host a relatively similar number of participants.

24 With the increasing demand for participation in future Symposia, space restrictions should be a key

25 consideration in selecting a venue. The total number of available sessions (after allocating ten non-

26 competitive slots to HSG's thematic working groups) (HSG) have been equally split between organized

27 sessions and individual abstract-driven sessions affording equal opportunity for both types. Consequently,

28 with the growing abstract submissions and fixing the number of sessions available, the acceptance rate for

29 the oral presentations is expected to decline, which was the case for HSR2018. Space availability in the

30 venue also constrains the number of posters that can be displayed. Poster space constraints can be relaxed

31 to some extent by having posters displayed for a single day, but this makes the organization of poster

32 judging more complex. The growing abstract submissions also incurs additional costs and time, limiting

33 the capacity of Symposia organizers to respond to the growing demand for engagement in the field.

34 Future research may also consider trends in attendance levels to the Symposia to better understand how 
1 increasing the number of abstracts accepted for presentation, as well as the use of bigger spaces, would

2 influence attendance and engagement of the Symposia participants.

4 To address the above concerns, Symposium organizers may consider additional and more diverse avenues

5 to stimulate the development of the HPSR field and its Symposia, such as media and online platforms,

6 and other spaces. Pursuing more versatile strategies can facilitate increasing engagement in the field and

7 allow more people to present at the event. Specifically, more or parallel venues can be added to

8 accommodate more parallel sessions; thus, increasing the total number of abstracts that can be presented

9 in the Global Symposia. Further, more region-specific Symposia and other online events can also be

10 organized, to respond to the growing demand of the researchers and other sectors to engage more in the

11 HPSR field and develop regional agendas. Given the increasing interest in presenting at global fora in

12 regions where HPSR is relatively new, there is a need for targeted support for institutions from these

13 regions, to increase abstract acceptance rate.

15 Overall, this study provides important baseline information to continuously and more comprehensively

16 track the field of HPSR. By using the online abstract submission system for the Global Symposia on

17 Health Systems Research, we provided additional information that is not yet captured in previous studies,

18 and that will allow to monitor the growth and expansion of HPSR. Existing data formats do not currently

19 allow for analysis of co-author's characteristics, as well as for thematic or topical analysis of abstracts and

20 research submitted for the Symposia. Once data become available and formatted to allow such analyses,

21 future work can explore the extent of HPSR collaborations and HSR agenda. Such information can also

22 track the extent of global collaborations in the HPSR field, which can then be supplemented with

23 additional data on engagement from existing HSG social networks. Further, abstract texts can also be

24 coded and examined to identify thematic trends within the field of HPSR, using qualitative methods. The

25 abstract texts would reveal additional information on study sites, which may be different from the country

26 affiliation of the authors. Their analysis can also identify the most common HPSR research topics and

27 focus areas across countries providing insights on country's research priorities. Making such information

28 available would reveal priority areas in HPSR based on country characteristics (e.g. country income

29 group, regions, institutional types) that can also be used in creating a taxonomy of HPSR topics.

\section{Limitations}


1 When interpreting the results, several limitations of the study have to be noted. First, the paper only

2 considers individual abstracts submitted for the two Symposia to indicate engagement in the field of

3 HPSR across various geographic regions, institutional types, and other author characteristics. The

4 Symposium programme also includes organized sessions and a rich pre-conference satellite programme.

5 The submission system did not capture data on individuals involved in either of these conference streams

6 as they have implicit bias towards selecting speakers for the session and these are therefore not included

7 in our analysis. Data on organized sessions are also not yet accessible in a format that can be used for

8 analysis. Future research work can include analysis of abstracts submitted for organized sessions once

9 data become available. Thus, the paper does not reflect the full range of individuals presenting at the

10 Symposium, which is better described in the Symposium external evaluation reports (HSG, 2017, 2019).

11 Second, our findings used characteristics of the corresponding authors, excluding characteristics of co-

12 authors (ranging from 1 to 37 authors for each submitted abstract). We were not able to use coauthor

13 information because coauthor characteristics were less complete compared to corresponding authors',

14 requiring significantly more manual data cleaning (e.g. addresses of co-author's institutional affiliations

15 were not as complete to be useful for geocoding of institutions) and better prediction tools (e.g. for gender

16 identification and institutional types). Since our data are de-identified, we cannot determine the number of

17 unique authors. Future studies may include analysis of the unique and co-authors once data and consent

18 for identifiable data become available. Our findings used predicted information on gender and

19 institutional types, which may not be as accurate compared to collecting data on these author

20 characteristics using the online abstract submission system. Global Symposia organizers may consider

21 enhancing the accuracy and completeness of the information about author characteristics, particularly

22 collecting gender information, to facilitate future studies. Another limitation of the study is that we do not

23 examine how the selection criteria have evolved throughout the years and how the composition of abstract

24 peer reviewers has changed, which could be a major factor for the symposia participant composition.

25 However, we assumed that this will not have a major influence on our findings because while the

26 reviewers may differ for the two Symposia, the selectionp criteria remained the same for both years.

27 Future studies can examine the influence of abstract peer reviewers and selection criteria once data

28 become available. Future Symposia may also request information on the position of the abstract authors to

29 distinguish abstracts that are from senior authors versus those that are from early career HPSR

30 participants.

31

32

\section{Conclusion}


1 The interest and engagement in the Global Symposia on HPSR has grown, with more people across

2 different sectors engaging and presenting their work in the Global Symposia, suggesting improved

3 inclusivity and diversity in HPSR. Engagement has increased across all sectors, in lower-income settings,

4 and in regions where HPSR capacity was noted to be weaker. Findings can be used to inform promotion

5 of inter-institutional collaborations across countries with high concentration of acceptances and those with

6 lower concentration of acceptances. Findings are also useful to guide the strategies directions of HSG in

7 ensuring that acceptance criteria consider the importance of welcoming researchers from under-

8 represented countries. Although our findings may suggest growing interest and engagement in the field,

9 more needs to be done to further advance HPSR globally. The reduction in acceptance rates from 2016 to

102018 also suggests that if the number of submissions continue to increase with the number of available

11 slots for presentation fixed, acceptance rates for the next Global Symposia are likely to continue to

12 decline. Such lower acceptance rates may limit inclusivity and diversity in HPSR field, requiring more

13 targeted strategies from Symposium organizers. These should be directed more towards further engaging

14 researchers and institutions from all sectors, especially those in lower-income setting and especially in

15 (sub)regions with weak HPSR capacity, and providing more avenues for participation and engagement in

16 the field of HPSR to accommodate the growing interest in the field. Therefore, the findings suggest a

17 challenging question for HSG - how to support the equitable growth of the HPSR field while managing

18 effectively significant organizational and logistical challenges, and what should be the future balance

19 between global, regional and virtual events to maximize the reach and diversity of individuals and

20 institutions involved in HPSR. 


\author{
Abbreviations: \\ HPSR Health Policy and Systems Research \\ HSS Health Systems Strengthening \\ SDGs Sustainable Development Goals \\ WHO World Health Organization
}

\title{
References
}

AHPSR. 2018. WHO: Alliance for Health Policy and Systems Research.

Bennett S, Agyepong IA, Sheikh K, Hanson K, Ssengooba F, Gilson L. 2011. Building the Field of Health Policy and Systems Research: An Agenda for Action. PLoS Medicine 8: e1001081.

Bennett S, Frenk J, Mills A. 2018. The evolution of the field of Health Policy and Systems Research and outstanding challenges. Health research policy and systems 16: 43.

Block MAG. 2006. The state of international collaboration for health systems research: what do publications tell? Health Research Policy and Systems 4: 7.

Genderize. 2018. Genderize.io. Genderize.io: Determine the gender of a first name.

Gilson L, Lehmann U, Schneider H. 2017. Practicing governance towards equity in health systems: LMIC perspectives and experience. International Journal for Equity in Health 16: 171.

Gonzalez-Block MA. 2004. Health policy and systems research agendas in developing countries. Health Research Policy and Systems 2: 6.

Gonzalez Block MA, Mills A. 2003. Assessing capacity for health policy and systems research in low and middle income countries*. Health Research Policy and Systems 1: 1.

Hafner T, Shiffman J. 2013. The emergence of global attention to health systems strengthening. Health Policy and Planning 28: 41-50.

HSG. Current Thematic Working Groups. https://healthsystemsglobal.org/twg-group/.

HSG. 2016a. Health Systems Global Strategic Plan 2016-2020

https://www.healthsystemsglobal.org/upload/other/HSG_StratReport_2016-2020.pdf

HSG. 2016b. Co-sponsors, Financial Contributors, and Partners | HSR2016.

https://healthsystemsresearch.org/hsr2016/co-sponsors-financial-contributors-partners-2/. Health Systems Global.

HSG. 2017. Evaluation of the Fourth Global Symposium on Health Systems Research. https://healthsystemsglobal.org/upload/other/Evaluation_HSR2016.pdf

HSG. 2018a. Co-sponsors and partners. http://healthsystemsresearch.org/hsr2018/co-sponsors-partners/.

\section{Health Systems Global2.}

HSG. 2018b. What makes a great organized session? HSR2018. 
http://healthsystemsresearch.org/hsr2018/news/makes-great-organized-session/.

HSG. 2018c. How Collectivity can help you to set up and shape your organized session.

http://healthsystemsresearch.org/hsr2018/news/collectivity-can-help-set-shape-organized-session/. Health Systems Global.

HSG. 2018d. HSG to co-host nine national meetings in Latin America ahead HSR2018.

https://www.healthsystemsglobal.org/blog/232/HSG-to-co-host-nine-national-meetings-in-LatinAmerica-ahead-HSR2018.html.

HSG. 2018e. Africa Region webinar: How to submit a successful organised session abstract.

https://www.healthsystemsglobal.org/webinars/30/Africa-Region-webinar-How-to-submit-asuccessful-organised-session-abstract.html.

HSG. 2019. Evaluation of the Fifth Global Symposium on Health Systems Research. https://www.healthsystemsglobal.org/upload/other/HSR2018-Final-Evaluation-report.pdf

Kraushaar D, Kieny M-P, Lazarus J V., et al. 2012. Health Systems Global, the new international society for health systems research. Health Policy and Planning 27: 535-40.

Python. 2018. Python.org. Python software.

Sheikh K, Bennett SC, el Jardali F, Gotsadze G. 2016. Privilege and inclusivity in shaping Global Health agendas. Health Policy and Planning 32: czw146.

Sheikh K, Gilson L, Agyepong IA, Hanson K, Ssengooba F, Bennett S. 2011. Building the Field of Health Policy and Systems Research: Framing the Questions. PLoS Medicine 8: e1001073.

Sundewall J, Swanson RC, Betigeri A, et al. 2011. Health-systems strengthening: current and future activities. The Lancet 377: 1222-3.

The Mexico Statement on Health Research. 2004.

WHO. 1996. Health policy and systems development : an agenda for research.

WHO. 2013a. The world health report 2000 - Health systems: improving performance. WHO.

WHO. 2013b. World health report 2013: Research for universal health coverage. WHO.

WHO. 2017. First World Report on Health Policy and Systems Research. WHO. 